\title{
nonsolo Verlag: Mut zu mehr Diversität im deutschen Verlagswesen - Courage for more Diversity in the German publishing Industry
}

\author{
Authors: Anna Munz \\ Submitted: $\quad$ 7. January 2021 \\ Published: $\quad$ 11. January 2021 \\ Volume: 8 \\ Issue: 1 \\ Affiliation: nonsolo Verlag, Freiburg, Germany \\ Languages: \\ Keywords: \\ Categories: \\ DOI: \\ German \\ Italy, Authors, Publishers, Courage, Diversity, Germany \\ News and Views, Humanities, Social Sciences and Law \\ 10.17160/josha.8.1.730
}

Abstract:

No doubt that articles written by Italian authors are represented on the German market. On closer inspection though, it becomes apparent that the focus is mostly on the well-known, big names. This leads to an outdated image about Italy and the Italian culture. nonsolo - a publisher based in Freiburg, Germany gives young Italian authors the chance to make their work know in the German market.

\section{JOSHA Jumualosianeas, Humanities and Arts}




\title{
nonsolo Verlag:
}

\section{Mut zu mehr Diversität im deutschen Verlagswesen - Courage for more diversity in the german publishing industry}

\author{
Anna Munz
}

\section{Abstract}

No doubt that articles written by Italian authors are represented on the german market. On closer inspection though, it becomes apparent that the focus is mostly on the well-known, big names. This leads to an outdated image about Italy and the Italian culture. nonsolo - a publisher based in Freiburg, Germany gives young Italian authors the chance to make their work know in the German market.

\section{Italien mal anders}

Werke italienischer Schriftsteller*innen sind auf dem deutschen Markt vertreten, keine Frage. Doch bei genauerem Hinsehen fällt auf, dass der Fokus meist auf den bereits bekannten, großen Namen liegt. Diese Dynamik des deutschen Verlagswesens ist nachvollziehbar. Sie bevorzugt Stimmen, die dem deutschen Lesepublikum bekannt sind, da diese einfacher den Weg auf den Markt finden. Jedoch führt dies dazu, dass ein Bild von Italien und von der italienischen Kultur entsteht, das teilweise veraltet ist und nicht mehr der aktuellen italienischen Lebensrealität entspricht. Dadurch wird zum einen den zeitgenössischen Autor*innen, die ein realistischeres Bild des heutigen Italien beschreiben, die Möglichkeit genommen, in Deutschland rezipiert zu werden, zum anderen werden der deutschsprachigen Leser*innenschaft bereichernde Lesemomente vorenthalten.

Dieser Tendenz entgegenzuwirken, hat sich der nonsolo Verlag, 2017 in Freiburg in Breisgau gegründet, zum Programm gemacht. Das durchweg zweisprachige kleine Team setzt seine volle verlegerische Aufmerksamkeit auf die Übersetzung ausschließlich zeitgenössischer italienischer Werke ins Deutsche. Dabei wählt es 
Autor*innen, die in Italien bereits für diverse Preise nominiert und auch ausgezeichnet wurden und dort eine gewisse Relevanz haben, die jedoch in der deutschsprachigen Literaturlandschaft bisher nicht wahrgenommen wurden. Bei der Auswahl der Werke legt die Verlegerin neben der hohen literarischen Qualität besonderen Wert darauf, dass der Text ein realistisches und diverses Bild des heutigen Italien zeichnet und dabei auch konfliktreiche Themen nicht ausspart.

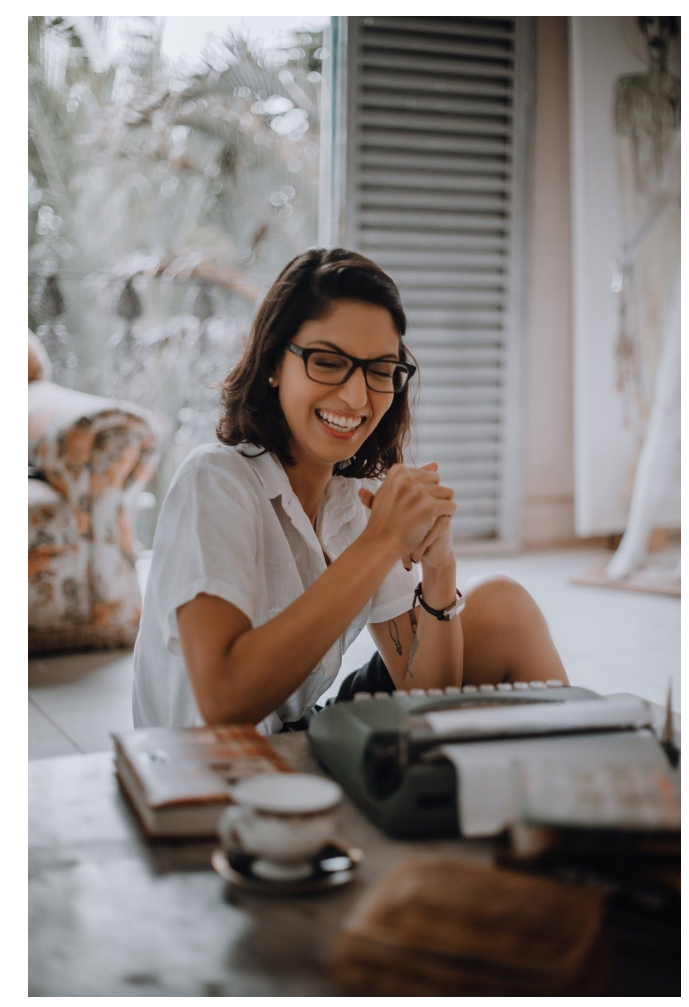

Dass dieses Vorhaben auf fruchtbaren Boden fällt, zeigt bereits das äußerst positive Echo der Öffentlichkeit und der Kritiker*innen, mit dem die zweisprachige Anthologie Spiegelungen/Vite allo specchio aufgenommen wurde, eine Sammlung von zehn unveröffentlichten Geschichten von ebenso vielen italienischen Autor ${ }^{\star} i n n e n$ (alphabetisch: Paolo Di Paolo, Simone Giorgi, Gabriella Kuruvilla, Gaia Manzini, Ludovica Medaglia, Demetrio Paolin, Anna Pavignano, Igiaba Scego, Simona Sparaco, Nadia Terranova), erschienen im Herbst 2018. Präsentiert wurde das Buch auf der Frankfurter und der Leipziger Buchmesse sowie live mit einzelnen oder auch allen Autoren gleichzeitig auf der Bühne in verschiedenen kulturellen Institutionen in Freiburg, Stuttgart, Hamburg, Berlin und Rom. 


\section{Übersetzungen auf höchstem Niveau}

Eine Besonderheit des Verlags ist das Verfahren der Übertragung des Textes ins Deutsche mit dem Ziel, dem Geist des Originals wirklich treu zu bleiben und alle Möglichkeiten der deutschen Sprache auszuschöpfen, um den Originaltext in all seinen Facetten dem hiesigen Lesepublikum zugänglich zu machen. Dabei geht es um viel mehr als um wortgetreue Übersetzungen. Es geht um das, was zwischen den Zeilen steht. Damit diese hohe Qualität erreicht werden kann, steht der Übersetzerin während des gesamten Übersetzungsprozesses eine Lektorin mit Muttersprache Italienisch zur Seite, Irene Pacini, die sie mit Rat und Tat unterstützt. $\mathrm{Zu}$ einer guten Übersetzung gehört manchmal eine gute Portion Mut. Genau darin besteht ihre Aufgabe: den Übersetzer*innen Mut zu machen, auch ungewöhnliche Lösungen auszuprobieren; als Muttersprachlerin, die den italienischen Text unmittelbar "fühlt" und gleichzeitig in der Lage ist, die möglichen deutschen Lösungen zu verstehen und zu bewerten. Selten geht es dabei um „richtig“ oder „falsch“, sondern vielmehr um die treffendste Annäherung an den Ausgangstext. Dieses Vorgehen leistet nicht nur einen wichtigen und hochwertigen Beitrag zur deutschen Literaturlandschaft, es ist ebenso ein Zeugnis für die bedingungslose Leidenschaft, mit der sich der Verlag der Verbreitung von italienischer Kultur und Literatur im deutschsprachigen Raum widmet.

Genau diesen Wert erkannte im November 2020 das italienische Ministerium für Kulturgüter, kulturelle Aktivitäten und Tourismus, als es dem nonsolo Verlag den Premio Nazionale per la Traduzione verlieh. Diesen erhalten üblicherweise einzelne Übersetzungen, dem nonsolo Verlag hingegen wurde die Anerkennung für seine gesamte verlegerische Tätigkeit zuteil, für die Sichtbarmachung und Verbreitung neuerer italienischer Literatur. In der Begründung betont das Ministerium die "lobenswerte Absicht der Verlegerin, ein echtes Pilotprojekt voranzutreiben. Der Preis geht an dieses mutige und verdienstvolle Unternehmen, auch im Hinblick auf eine produktive und erfolgreiche Zukunft."

Die Stimmen des Verlags 


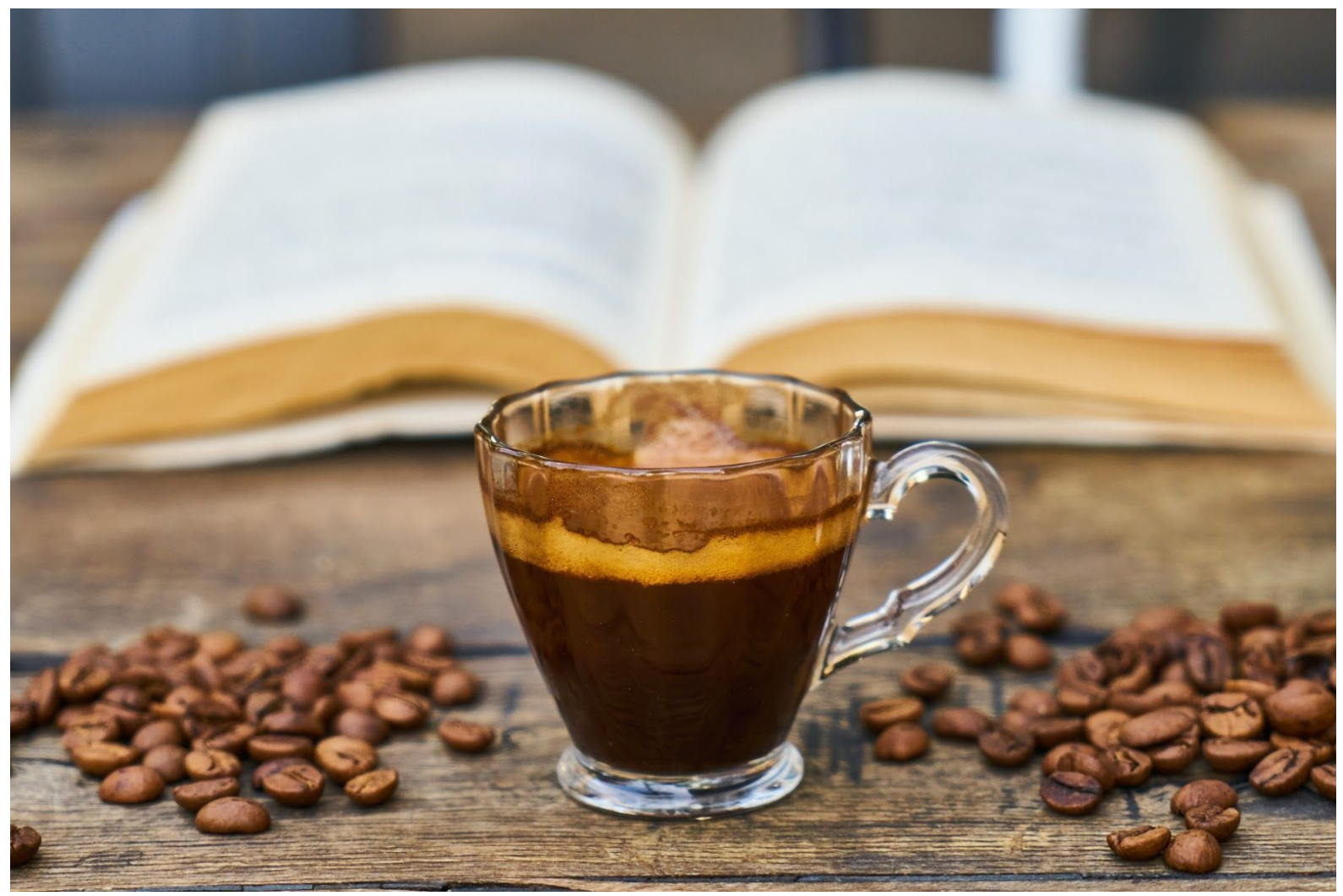

Nach der zweisprachigen Anthologie, die als Debüt auf dem deutschen Markt als eine Art Vorankündigung für das folgende Programm fungiert, erschienen bisher zwei Romane und eine weitere Anthologie in der neu gegründeten Reihe nonsolo mini.

Im Mai 2019 erschien der Roman Hummerjahre von Nicola H. Cosentino ausgezeichnet mit dem Premio Brancati Sezione Giovani 2018, übersetzt von Ruth Mader-Koltay, eine in mehreren Episoden dargestellte Freundschaft zwischen zwei jungen Männern von der Pubertät bis zum Erwachsenwerden. Die großen Themen des Lebens werden dabei wie zufällig aufgegriffen und auf sehr authentische und nachfühlbare Art in die Lebenshaltung der jungen Männer eingebunden: Die Herausforderungen einer Freundschaft, die Suche nach Heimat, nach der eigenen Bestimmung im Leben, Tod und Trauer, Elternwerden und immer und immer wieder die verzwickten Probleme, die die Liebe mit sich bringt, begleiten die Protagonisten über mehrere Jahre.

Der Roman Fast nur eine Liebesgeschichte von Paolo di Paolo erschien im Dezember 2019 in der Übersetzung von Christiane Burkhardt. Der Autor, mit zahlreichen Preisen in Italien ausgezeichnet und 2013 nominiert für den Premio Strega, den wichtigsten italienischen Literaturpreis, präsentiert mit diesem Roman ein vielschichtiges Werk, das auf beinahe philosophische Weise dazu anregt, über die Kunst und das Leben, über die Jugend und das Altern nachzudenken. Die Liebesgeschichte zwischen den ungleichen Figuren Nino und Teresa wird aus der 
Perspektive einer dritten Person, Teresas Tante und Ninos Mentorin, erzählt und ermöglicht so eine Erweiterung der eigentlichen Erzählung um eine zusätzliche, spannende Ebene.

Die vierte Publikation des Verlags begründet eine zweite Reihe innerhalb des Verlagsprogramms, nonsolo mini, und richtet sich damit unter anderem an Universitäten und Schulen. Die Anthologie Dismatria und weitere Texte von Igiaba Scego, übersetzt von Ruth Mader-Koltay, erschien im September 2020. Neben der titelgebenden Kurzgeschichte Dismatria, die bis zum Jahr 2024 Sternchenthema des baden-württembergischen Abiturs im Fach Italienisch ist, enthält das Buch eine weitere Erzählung und einen Artikel der Autorin und ein ausführliches kritisches Vorwort von Prof. Martha Kleinhans (Universität Würzburg), Expertin für transkulturelle Literatur. Igiaba Scego, Italienerin somalischer Abstammung, beschäftigt sich in ihren Texten mit der Migration, der Sehnsucht nach Heimat, mit Entwurzelung und Identitätssuche. Auf witzige und selbstironische Art macht sie diese schwierigen Themen ihren Leser*innen zugänglich und erzählt so von einem neuen, diversen Italien.

Für das Frühjahr 2021 sind zwei Publikationen geplant: Der Roman Ohne Halt ins Blaue von Anna Pavignano, einer renommierten Drehbuchautorin und Schriftstellerin, übersetzt von Ruth Mader-Koltay, beschreibt die konfliktbeladene Lebensrealität eines jungen Mannes, der im Sommer auf seiner Heimatinsel Touristenausflüge auf seinem Boot anbietet und im Winter eine ganz andere Identität annimmt, wenn er zur Arbeit auf der Baustelle aufs Festland übersiedelt. Eine Geschichte von der Liebe und dem Meer, von Freundschaft und Tragödien, von dem Konflikt zwischen zwei Kulturen, auf ironische und leichte Weise geschrieben.

Außerdem wird im Frühjahr 2021 in der Reihe nonsolo mini das Theaterstück Fünf Frauen. Lebensgeschichten aus Süditalien/Cinque donne del sud von Francesca Zanni in einer zweisprachigen Fassung erscheinen. In den fünf Geschichten der Protagonistinnen spiegelt sich symbolhaft die bewegte Geschichte Italiens seit der politischen Einigung Ende des 19. Jahrhunderts wider, bis hin zur selbstbewussten Generation der Digital Natives, die zu ihren Wurzeln zurückfindet. 


\section{Die Herausforderungen unserer Zeit}

Eine Herzensangelegenheit des Verlags ist es, nicht nur die Werke der gewählten Autor*innen im deutschsprachigen Raum zu verbreiten, sondern die Schriftsteller*innen persönlich nach Deutschland zu holen. So legt der Verlag in seiner Marketingstrategie besonderen Wert auf Lesungen und Veranstaltungen, bei denen es dank der Begleitung einer Übersetzerin auch rein deutschsprachigen Leser*innen möglich ist, mit den Autor*innen direkt ins Gespräch zu kommen. Es liegt auf der Hand, dass die pandemiebedingte Lahmlegung des kulturellen Lebens seit dem Frühjahr 2020 einen herben Rückschlag für den Verlag bedeutet. Die meisten Veranstaltungen mussten auf unbestimmte Zeit verschoben, Präsentationen auf den Buchmessen abgesagt werden. Einige Lesungen und Seminare an Schulen und Universitäten fanden und finden auch zukünftig digital statt, jedoch können sie ein persönliches Treffen nicht ersetzen. Der Ausblick auf das Jahr 2021, das mit einem erneuten Lockdown beginnt, ist ungewiss, doch das Team von nonsolo Verlag ist überzeugt: Das Projekt lohnt sich, und irgendwann werden wir alle wieder zu einem unbeschwerten Leben zurückkehren können. Bis dahin heißt es optimistisch bleiben und jede sich bietende Gelegenheit wahrnehmen, das Ziel, eine Brücke zwischen der deutschen und der italienischen Kultur und Literatur zu schlagen, auf alternativen Wegen zu erreichen.

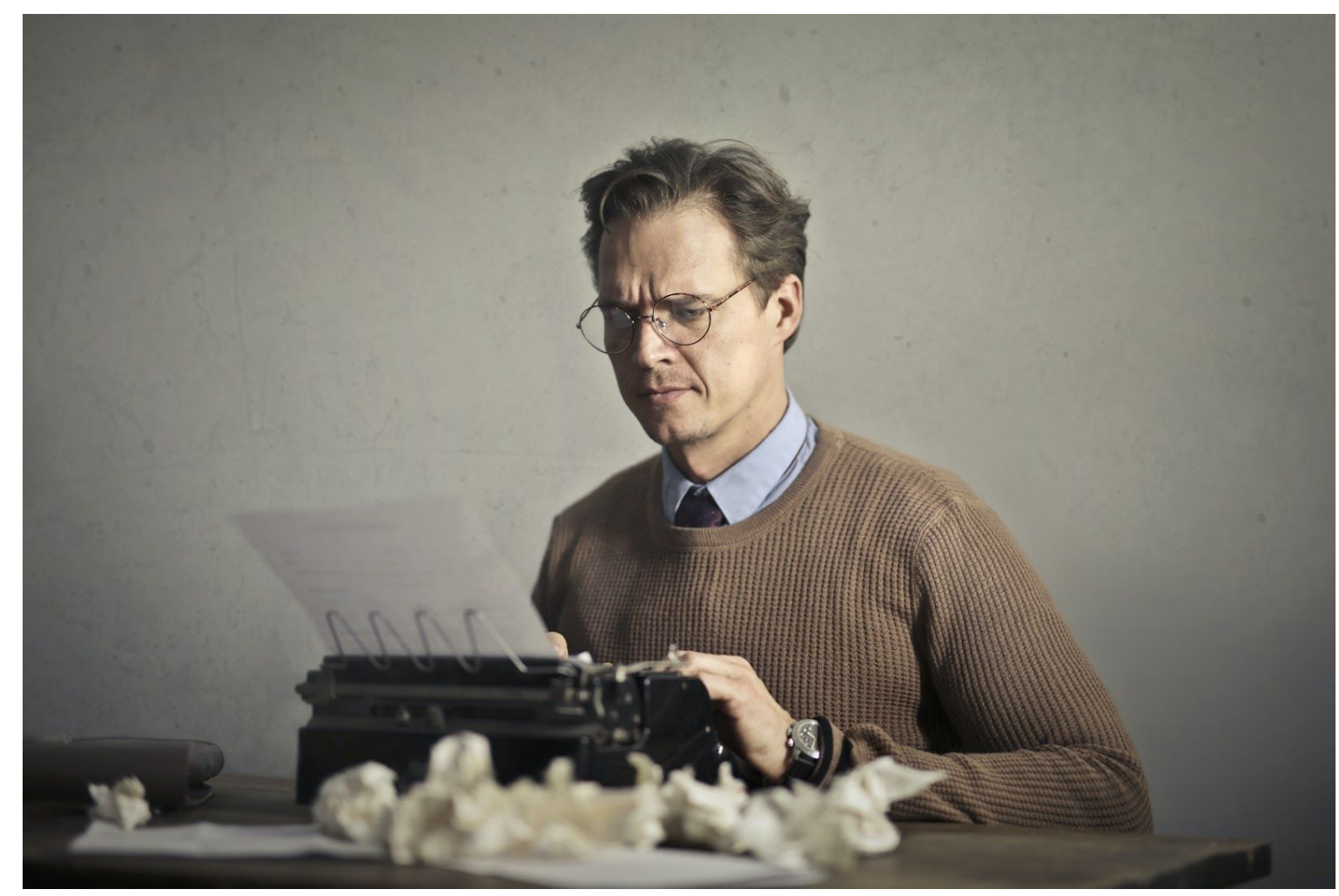

